\title{
Metastatic Gallbladder Carcinoma in Meningioma: A Case Report
}

\author{
Burak EREN, Feyza KARAGOZ GUZEY, Ilker GULEC, Azmi TUFAN \\ Health Sciences University, Bagcilar Training and Research Hospital, Neurosurgery Clinic, Istanbul, Turkey \\ This study has been presented an oral presentation in the $30^{\text {th }}$ Turkish Neurosurgical Society Congress between 8 and 12 April, 2016 at Antalya, Turkey.
}

Corresponding author: Burak EREN drburakeren@hotmail.com

\section{ABSTRACT}

Metastases from tumors to systemic cancers are rare. The most common intracranial recipient tumor is meningioma. Metastasis from gallbladder cancer has been previously reported from only one patient during autopsy. We present a case of a 72-year-old woman who underwent surgery for right frontal skull base meningioma. The tumor was completely removed. Histological specimens showed gallbladder carcinomatous metastasis with diffuse neuroendocrine differentiation in meningothelial meningioma. The Ki-67 proliferation index of the meningioma was $3 \%$. Further, $60 \%$ positive immunoreactivity with the progesterone receptor was observed in meningioma cells. In carcinoma cells, diffuse positive immunoreactivity with chromogranin, CDX2, CEA, panCK, cytokeratin 7, and synaptophysin was observed. A combination of molecular, metabolic, immunological, and/or hormonal factors may contribute to the pathogenesis of this lesion. It cannot be ruled out that it is more common than expected.

KEYWORDS: Gallbladder carcinoma, Histopathological examination, Meningioma, Metastasis into meningioma

\section{INTRODUCTION}

$\mathrm{M}$ etastases to systemic cancers are rare in tumors (14). The most common intracranial recipient tumor is meningioma (4). Most common sources of metastases into meningiomas are breast and lung cancers; metastases from renal, thyroid, prostate, and colon cancers are very rare $(1,13)$, and those from gallbladder cancer have been observed in tissue sections obtained during autopsy from one patient (11). Molecular, hemodynamic, hormonal, and metabolic hypotheses may explain intratumor metastasis; however, the main mechanism remains unclear.

We present a patient who underwent surgery for meningioma and was diagnosed with gallbladder carcinoma after histopathological examination.

\section{CASE REPORT}

A 72-year-old female patient presented to our clinic with numbness of the left side for a month. Neurological examina- tion showed hemiparesis. She had a history of chronic hypertension and glaucoma and had undergone right nephrectomy for renal clear cell carcinoma 10 years previously. She was followed up by the medical oncology clinic for 5 years and showed no recurrence. Brain magnetic resonance imaging (MRI) performed 3 years previously showed no intracranial pathology. A new MRI revealed a $4.5 \times 4 \times 3.5-\mathrm{cm}$ meningioma on the right frontal skull base (Figure 1). The tumor was completely removed. Her postoperative general condition was good; she was discharged on postoperative day 5 after normal neurological examination findings.

Surgical specimens were fixed in $10 \%$ formalin for light microscopy. Selected samples from case specimens were routinely embedded in paraffin, and 5 - $\mu$ m-thick sections were cut and stained using hematoxylin and eosin (H\&E). Immunohistochemistry using specific antibodies was performed on paraffin-embedded tissue sections (Figure 2). Histological specimens showed gallbladder carcinomatous metastasis with diffuse neuroendocrine differentiation in meningothelial
Burak EREN (D): 0000-0001-5554-2585

Feyza KARAGOZ GUZEY (1) : 0000-0002-4260-9821
Ilker GULEC (D) : 0000- 0003- $4207-238 X$

Azmi TUFAN (1) : 0000-0001-9042-8542 

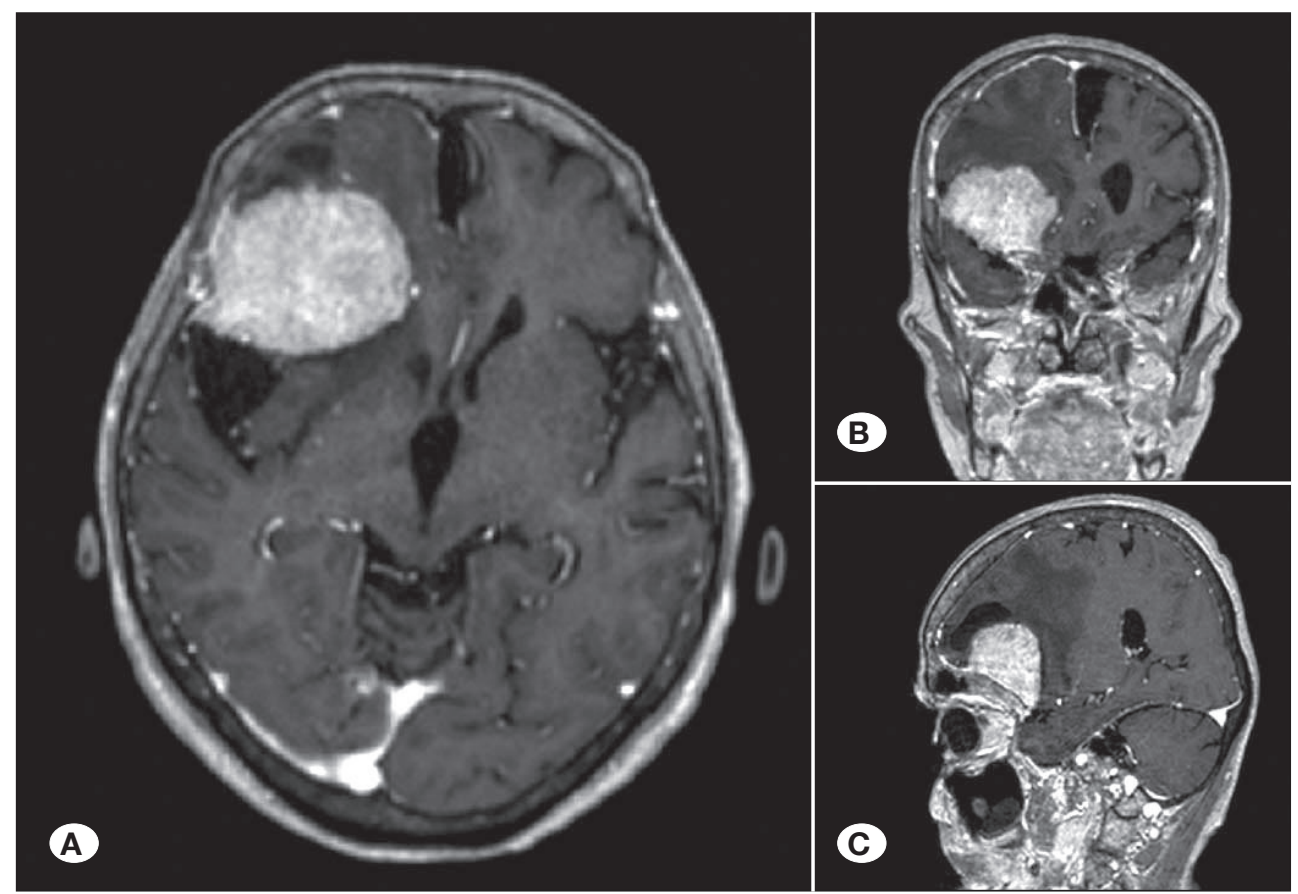

Figure 1: Axial (A), coronal (B), and sagittal (C) T1W contrast enhanced MRI scans. Heterogeneous enhancement of $4.5 \times 4 \times 3.5 \mathrm{~cm}$ meningioma located on right frontal skull base was observed.

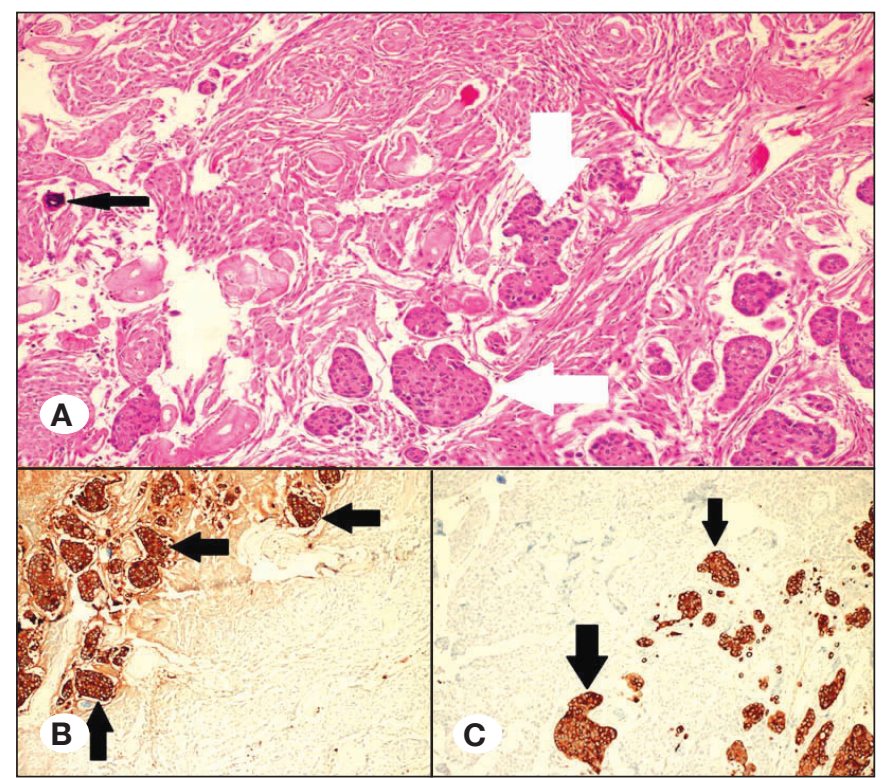

Figure 2: A) Psammomatous calcification (black arrow) on the left and carcinoma infiltration (white arrows) on the right and lower solid epithelial islands are seen in middle hyaline-like turbulence structures formed by meningothelial cells (H\&E, $\times 100)$. B) Immunoreactivity (black arrows) was observed in the carcinoma areas (panCK, $\times 100)$. C) Immunoreactivity was observed in carcinoma cells (black arrows) (CEA, $\times 100)$.

meningioma. The Ki-67 proliferation index of the meningioma was $3 \%$. Further, $60 \%$ positive immunoreactivity with the progesterone receptor was observed in meningioma cells. In carcinoma cells, diffuse positive immunoreactivity with chromogranin, CDX2, CEA, panCK, cytokeratin 7, and synapto- physin was observed. No staining was observed with CK20 and TTF-1.

One day before the pathological report, she presented to our hospital's emergency department with severe abdominal pain. Neurological examination findings were normal; no nuchal stiffness was observed. Brain computed tomography (CT) findings were normal. No neurosurgical pathology was found to explain her condition. She had acute abdominal findings. Her condition deteriorated following radiological examinations, and she developed sudden cardiac arrest. She did not respond to resuscitation. One day later, she was diagnosed with gallbladder cancer. Preoperative abdominal CT images were reviewed, and a new mass was observed on the gallbladder.

\section{DISCUSSION}

Tumor-to-tumor metastasis is rare and was first described in 1930 (5). Among all benign tumors, meningiomas have the highest rate of metastasis from primary tumors (6). All reported intrameningioma metastases are associated with intracranial meningioma, except for a few cases of spinal meningioma $(10,13)$. The most common metastatic sources are breast and lung carcinomas (4). Other malignancies exceptionally metastasize into meningiomas (e.g., renal, genitourinary, gastrointestinal, prostate, and parotid tumors and lymphoma) (12). Meningiomas such as breast cancers are twice as common in women than in men and occur at 50-60 years of age (4). Meningiomas are highly vascular tumors that sensitize hematological metastases. High collagen and lipid contents can be suitable for the growth of other tumors (15).

Tumorigenic factors such as mutation or deletion of tumor suppressorgenes may fall under the associationand/or affinities 
between metastatic breast carcinoma and meningioma. Also in meningioma patients, a depressive systemic cellular immune response may weaken the host's surveillance of metastasis (9). The presence of three tumors (renal clear cell carcinoma, meningioma, and gallbladder carcinoma) may support the hypothesis that tumorigenic factors were present in our patient. Hormonal factors may cause meningioma metastasis of breast carcinoma. Meningiomas have $83 \%$ progesterone and $9 \%$ estrogen receptors (7). Our patient had $60 \%$ positive immunoreactivity with the progesterone receptor.

Correct diagnosis of lesions before surgical removal and histological examination of meningiomas is almost impossible (2). Routine brain imaging with CT and MRI cannot exclude intrameningiomatous metastasis; hence, physiologically based brain imaging methods such as magnetic resonance spectroscopy (sMRI) and perfusion MRI (pMRI) have been proposed (4). The ratio of choline/creatinine in the sMRI can indicate the proliferative or malignant potential of meningioma, as well as the lactate/lipid peak associated with intratumoral necrosis (9). Watanabe et al. reported a case of breast cancer metastasis into a frontal parasagittal meningioma (15). Preoperative sMRI suggested the malignant nature of the tumor. However, definitive diagnosis was provided by histology.

Although meningiomas show characteristic findings, a faster than expected growth suggests that they may originate from metastatic disease (3). A study correlating meningioma growth with histology showed a mean doubling time of 415 days (range, 138-1045 days) in benign, 178 days (34-551 days) in atypical, and 205 days (30-472 days) in anaplastic meningiomas (8). Brain MRI of our patient obtained 3 years previously did not show a tumor, but the appearance of a 4-cm tumor in the new MRI suggested a short doubling time. Although the meningioma was histologically grade I, hypointense areas within the tumor were observed in MRI findings. Tumor-to-tumor metastasis may be suspected in meningiomas that are not homogeneous on MRI and have a short doubling time, as in our case.

Metastasis to meningiomas may clinically and histopathologically mimic metastatic disease. Generally, these patients present with meningioma-related complaints rather than with metastatic cancer findings. Clinically, various signs and symptoms may occur (13). Our patient was referred with hemiparesis.

The clinical significance and prognostic implication of this pathological condition are uncertain. Future investigations should clarify whether death is mainly due to meningioma or metastatic primary cancer. We believe that our patient died of gallbladder cancer, but it was not diagnosed because she did not undergo an autopsy.

\section{- CONCLUSION}

Most metastases are incidentally recorded during an operation or autopsy. A combination of molecular, metabolic, immunological, and/or hormonal factors may contribute to the pathogenesis of this lesion. It cannot be ruled out that this phenomenon is more common than expected.

\section{REFERENCES}

1. Aghi M, Kiehl TR, Brisman JL: Breast adenocarcinoma metastatic to epidural cervical spine meningioma: Case report and review of the literature. J Neurooncol 75:149-155, 2005

2. Baratelli GM, Ciccaglioni B, Dainese E, Arnaboldi L: Metastasis of breast carcinoma to intracranial meningioma. J Neurosurg Sci 48:71-73, 2004

3. Bhojwani N, Huang J, Gupta A, Badve C, Cohen ML, Wolansky LJ: Rectal carcinoid tumor metastasis to a skull base meningioma. Neuroradiol J 29(1):49-51, 2016

4. Caroli E, Salvati M, Giangaspero F, Ferrante L, Santoro A: Intrameningioma metastasis as first clinical manifestation of occult primary breast carcinoma. Neurosurg Rev 29(1):49-54, 2006

5. Fried BM: Metastatic inoculation of a meningioma by cancer cells from a bronchiogenic carcinoma. Am J Pathol 6:47-52, 1930

6. Honma K, Hara K, Sawai T: Tumour-to-tumour metastasis. A report of two unusual autopsy cases. Virchows Arch A Pathol Anat Histopathol 416:153-157, 1989

7. Hsu DW, Efird JT, Hedley-Whyte ET: Progesterone and estrogen receptors in meningiomas: Prognostic considerations. J Neurosurg 86:113-120, 1997

8. Jääskeläinen J, Haltia M, Laasonen E, Wahlström T, Valtonen $\mathrm{S}$ : The growth rate of intracranial meningiomas and its relation to histology. An analysis of 43 patients. Surg Neurol 24:165172, 1985

9. Lanotte M, Benech F, Panciani PP, Cassoni P, Ducati A: Systemic cancer metastasis in a meningioma: Report of two cases and review of the literature. Clin Neurol Neurosurg 111: 87-93, 2009

10. Okada E, Nakamura M, Koshida Y, Mukai K, Toyama Y, Matsumoto M: Breast carcinoma metastasis to meningioma in the thoracic spine: A case report and review of the literature. J Spinal Cord Med 38(2):231-235, 2015

11. Peison WB, Feigin I: Suprasellar meningioma containing metastatic carcinoma. Report of case. J Neurosurg 18:688689, 1961

12. Ravnik J, Ravnik M, Bunc G, Glumbic I, Tobi-Veres E, Velnar T: Metastasis of an occult pulmonary carcinoma into meningioma: A case report. World J Surg Oncol 13:292, 2015

13. Sayegh ET, Burch EA, Henderson GA, Oh T, Bloch O, Parsa AT: Tumor-to-tumor metastasis: Breast carcinoma to meningioma. J Clin Neurosci 22(2):268-274, 2015

14. Schmitt HP: Metastases of malignant neoplasms to intracranial tumours: The "tumour-in-a-tumour" phenomenon. Virchows Arch (Pathol Anat) 405:155-160, 1984

15. Watanabe T, Fujisawa H, Hasegawa M, Arakawa Y, Yamashita $\mathrm{J}$, Ueda F, Suzuki M: Metastasis of breast cancer to intracranial meningioma: Case report. Am J Clin Oncol 25:414-417, 2002 